\title{
Accuracy of Contrast-Enhanced Ultrasound in the Diagnosis of Bile Duct Obstruction
}

Authors

Affiliations
F. J. P. Fontán ${ }^{1}$, Á. R. Reboredo ${ }^{2}$, A. R. Siso

${ }^{1}$ Radiology, Complejo Hospitalario Universitario de A Coruña, A Coruña, Spain

${ }^{2}$ Radiology, Complejo Hospitalario Universitario Arquitecto Marcide, A Coruña, Spain
Key words

- abdomen

biliary system

ultrasound received $\quad 03.03 .2015$

accepted 22.06.2015

\section{Bibliography}

DOI http://dx.doi.org/ $10.1055 / \mathrm{s}-0035-1555880$

Published online: 2015

Ultrasound International Open

2015; 1: E12-E18

(c) Georg Thieme Verlag KG Stuttgart · New York

ISSN 2199-7152

\section{Correspondence}

\section{Dr. Ángel Ríos Reboredo}

Radiology

Complejo Hospitalario Universitario Arquitecto Marcide

Avenida Residencia, s/n

15405 Ferrol

A Coruña

A Coruña

15009 Spain

Tel.: +34/658/144 156

Angel.Rios.Reboredo@sergas.es

\section{License terms}

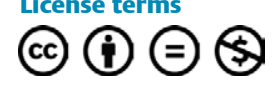

\section{Abstract}

$\nabla$

Purpose: To investigate the ability of contrastenhanced ultrasound (CEUS) to differentiate benign from malignant lesions causing biliary duct obstruction.

Materials and Methods: Between November 2006 and December 2013, 59 patients with bile duct obstruction of undetermined cause in baseline ultrasound underwent CEUS study. The enhancement and posterior washout were analyzed in real time all along the study duration $\left(5^{\prime}\right)$. The final diagnosis suggested by CEUS was compared with histologic diagnosis (47.5\%) or with radiologic follow-up with TC, RM or ERCP. Results: Final diagnoses included 42 malignant lesions (cholangiocarcinoma $n=22$, metastases $n=6$, pancreatic carcinoma $n=6$, hepatocarci-

\section{Introduction}

$\nabla$

Conventional ultrasound is the first-line imaging method for investigating biliary diseases. Once bile duct obstruction is detected, it is necessary to determine both the stop level and its nature. Baseline sonography may be able to localize the intraor extrahepatic origin of cholestasis but, in general, has a relatively low accuracy for this purpose $[1,2]$. As a consequence, in many cases it is necessary to combine ultrasound (US) with other noninvasive methods, including computed tomography (CT) or magnetic resonance (MR), or invasive imaging techniques, including endoscopic retrograde cholangiopancreatography (ERCP) or echo-endoscopy, to get the final diagnosis.

Development of new sonographic contrast media and improvements in detection techniques with low acoustic power have represented significant advances in ultrasound diagnosis. Second-generation ultrasound contrast agents include gas microbubble-based substances, stabilized by a noma $n=4$, gallbladder carcinoma $n=2$, ampullary carcinoma $\mathrm{n}=1$ and lymphoma $\mathrm{n}=1$ ) and 17 benign lesions (lithiasis or biliary sludge $n=15$, xanthogranulomatous cholecystitis $\mathrm{n}=1$ and indeterminate $n=1$ ). CEUS accuracy compared with final diagnoses based on combined reference standard was $86.4 \%$. CEUS correctly identified 36 of 42 malignant lesions (sensibility $85.7 \%$ ) and 15 of 17 benign lesions (specificity $88.2 \%$ ). The positive predictive value of CEUS for malignancy was $94.7 \%$, while the negative predictive value was $71.4 \%$.

Conclusion: CEUS is useful to differentiate between benign and malignant causes of obstructive jaundice. This technique improves the detection of bile duct invasion in hepatic neoplasms and permits better evaluation of intra- and extraductal extension of hilar hepatobiliary tumors.

biocompatible thin shell, usually of lipid compo-

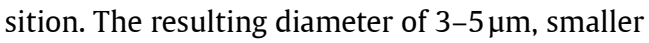
than red cells, is still much bigger than the particles used with CT and MR. For this reason, the ultrasound contrast is confined to the intravascular space, and does not cross to the extracellular milieu, facilitating a thorough study of microand macrocirculation in the target lesion [3]. Other advantages of contrast-enhanced ultrasound (CEUS) over CT and MR include its hypoallergenic, non-nephrotoxic conditions [4], as well as great versatility, because it can be performed in almost any setting, involves no radiation and permits real-time dynamic study of enhancement.

The utility of CEUS in the diagnosis of liver tumors is widely accepted. The European Federation of Societies of Ultrasound in Medicine and Biology (EFSUMB) published the first guide of clinical practice for this technique in 2004 [5]. More recently, the use of intravascular and intracavitary CEUS in different organs has resulted in 
new EFSUMB guidelines for non-hepatic use in 2008. A more recent update (2011) has recognized the usefulness of CEUS for the diagnosis of biliary diseases [6].

There are few studies in the literature exploring the benefits of CEUS in biliary diseases [7-9]. Moreover, all of them evaluate its use in gallbladder and biliary duct disease from a general point of view, but do not investigate its diagnostic value in biliary duct obstruction. The aim of this study was to determine the usefulness of CEUS to differentiate among benign and malignant biliary duct obstruction.

\section{Materials and Methods}

\section{Study population}

Between November 2006 and December 2013, 59 patients (33 men and 26 women) underwent biliary CEUS under the following requisites: 1 . intrahepatic (duct diameter $>40 \%$ above that of the adjacent portal vein) or extrahepatic ( $>7 \mathrm{~mm}$ ) bile duct dilatation demonstrated by baseline ultrasound in a patient with clinical findings of obstructive jaundice, 2. Unknown cause of biliary duct obstruction. Patients were excluded if the diagnosis was straightforward after baseline ultrasound, as in cases of lithiasis with acoustic shadow or masses in the pancreatic head. We also excluded patients with known severe cardiopathy and pregnant or lactating women. The study fulfilled the ethical rules of our institution. In our unit, contrast-enhanced ultrasound is routinely indicated in the case of a suspected neoplastic lesion. Considering that the study protocol did not require any further invasive maneuvers, we did not request formal approval by the local ethical committee of our center. Patients were specifically informed of the nature and conditions of the ongoing study and verbal informed consent was requested and obtained in all cases.

We used the histologic reports from samples obtained by ERCP or surgery as the reference diagnosis. When these were not available, we used clinical and radiologic follow-up data provided by CT, MRI or ERCP. Lesions were classified as benign, malignant or indeterminate, according to these results.

\section{Ultrasound technique}

All ultrasound examinations were performed by the same radiologist with more than 8 years of CEUS experience. In all studies baseline US examinations were the first approach. After confirmation of bile duct obstruction, a bolus of $2.5 \mathrm{ml}$ of Sonovue ${ }^{\circledR}$ (Bracco, Milan, Italy), composed by sulfur hexafluoride microbubbles with a phospholipid shell, was injected through the antecubital vein, and CEUS study was performed in real time along 5', with a low acoustic power contrast-specific imaging technique. A simultaneous display of tissue and contrast signals ensured visualization of the dynamic enhancement and washout patterns. Relevant images and videos were recorded digitally.

\section{Data analysis}

Images were interpreted initially by a single radiologist, with more than 8 years of CEUS experience, who was not aware of the results of the other imaging test. Subsequently, an interpretative consensus was reached by all authors after revisiting images and videos. Basal ultrasound was analyzed and then compared with relevant CEUS findings. Pathologic and imaging studies were recorded in a database, and the same applied for the anatomic locations of the lesions in the biliary tree (intrahepatic, hilar, common hepatic duct, common biliary duct and intrapancreatic duct). Sensitivity was calculated as the fraction of true positives over the number of true positives plus false negatives, i.e., the total number of malignant lesions, as demonstrated by the combined reference standard. Specificity was calculated as the fraction of true negatives over the number of true negatives plus false positives. Diagnostic accuracy was defined by the number of true positives plus true negatives divided by the total number of patients. Positive predictive value was calculated by dividing the true positives by the total positive results in CEUS. Negative predictive value was calculated by dividing the true negatives by the total negative results in CEUS.

\section{Results}

$\nabla$

CEUS was performed in 59 patients ( 33 men and 26 women, with a mean age of 71.5 years (range 48-90)). The final diagnoses included 42 malignant and 17 benign lesions. A histologic analysis was possible in 28 patients (47.5\%), while the diagnosis was based on data from the clinical and radiological follow-up in the remaining 31 cases (52.5\%) ( $\bullet$ Table $\mathbf{1}$ ). $\bullet$ Table 2 shows the final diagnoses.

Among malignancies, the most frequent diagnosis was cholangiocarcinoma (22 patients). In 6 cases of hilar cholangiocarcinoma, baseline ultrasound disclosed intrahepatic bile duct dilatation with possible hilar lesions, isoechoic to liver parenchyma. CEUS demonstrated in all cases infiltrating enhancing lesions in the biliary confluence with later washout and discrete margins ( $\bullet$ Fig. 1).

Extrahepatic cholangiocarcinoma presented as common hepatic duct lesions in 7 cases and common biliary duct lesions in 9 cases; all but one of these cases showed focal parietal thickening or intraductal lesions of variable echogenicity. CEUS demonstrated hyperenhancement and posterior washout in intraductal lesions, pointing to its tumoral nature ( $\boldsymbol{\bullet} \mathbf{~ F i g . ~} \mathbf{2 , 3}$ ). In one case CEUS showed a hypoechoic non-enhancing completely necrotic mass with portal malignant thrombosis (० Fig. 4).

Table 1 Standard of reference for the final diagnosis of biliary lesions.

\begin{tabular}{lc} 
Diagnostic technique & Number of lesions (\%) \\
Pathologic analysis & $28(47.5)$ \\
\hline Contrast-enhanced CT & $35(59.3)$ \\
Contrast-enhanced MR & $25(42.4)$ \\
ERCP & $28(47.4)$
\end{tabular}

Table 2 Final diagnosis of biliary lesions (total $=59$ ).

\begin{tabular}{|lc}
\hline Final diagnosis & Number \\
Benign & 17 \\
\hline Biliary sludge/gallstones & 15 \\
\hline Xanthogranulomatous cholecystitis & 1 \\
\hline Inconclusive & 1 \\
Malignant & 42 \\
\hline Cholangiocarcinoma & 22 \\
\hline Metastases & 6 \\
\hline Pancreatic carcinoma & 6 \\
\hline Hepatocellular carcinoma & 4 \\
\hline Gallbladder carcinoma & 2 \\
\hline Ampullary carcinoma & 1 \\
\hline Lymphoma & 1
\end{tabular}



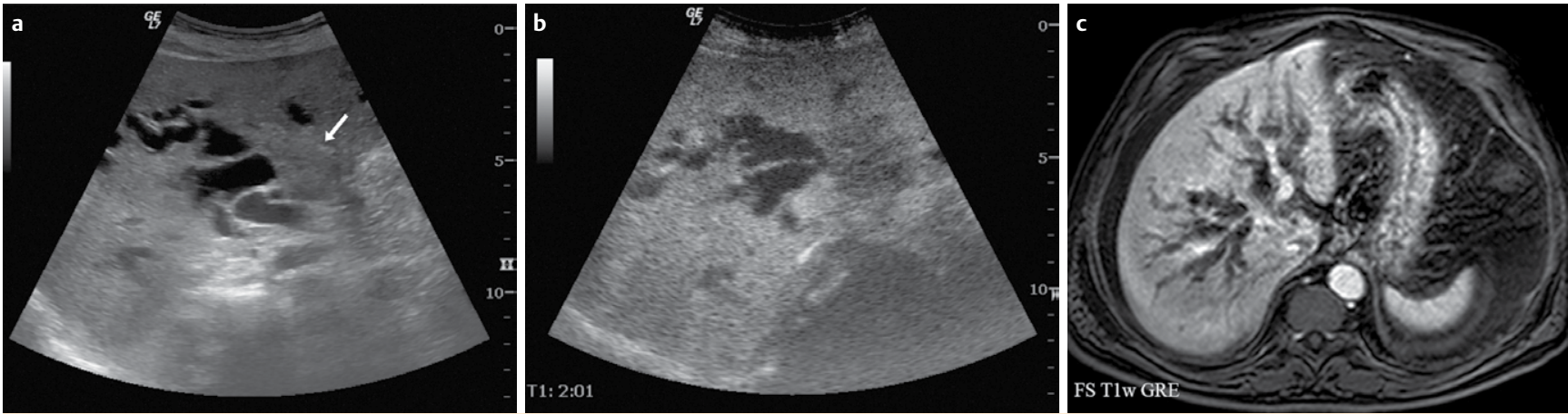

Fig. 1 Klatskin tumor. Baseline ultrasound a shows intrahepatic bile duct dilatation with hilar mass isoechoic to liver parenchyma (arrow). The lesion showed isoenhancement in the arterial and portal phase (not shown), but in the late phase CEUS improves the definition of the mass and its limits, showing a discrete hypoechoic mass 2 min after contrast agent injection in the washout phase $\mathbf{b}$. Dynamic contrast-enhanced MR in the late phase shows biliary duct dilatation but fails to improve lesion definition $\mathbf{c}$.
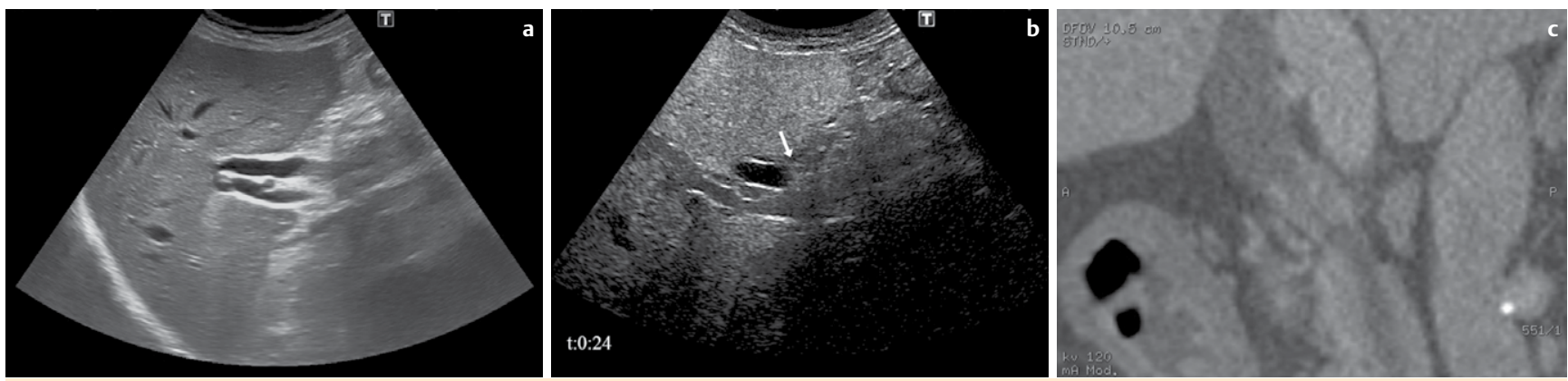

Fig. 2 Extrahepatic cholangiocarcinoma. Baseline ultrasound shows mild common bile duct dilatation filled distally with a hyperechoic cast a. CEUS shows cast enhancement with moderate washout in later phases $\mathbf{b}$. Coronal reformatted $\mathrm{CT}$ image nicely shows the neoplastic ductal wall thickening $\mathbf{c}$.
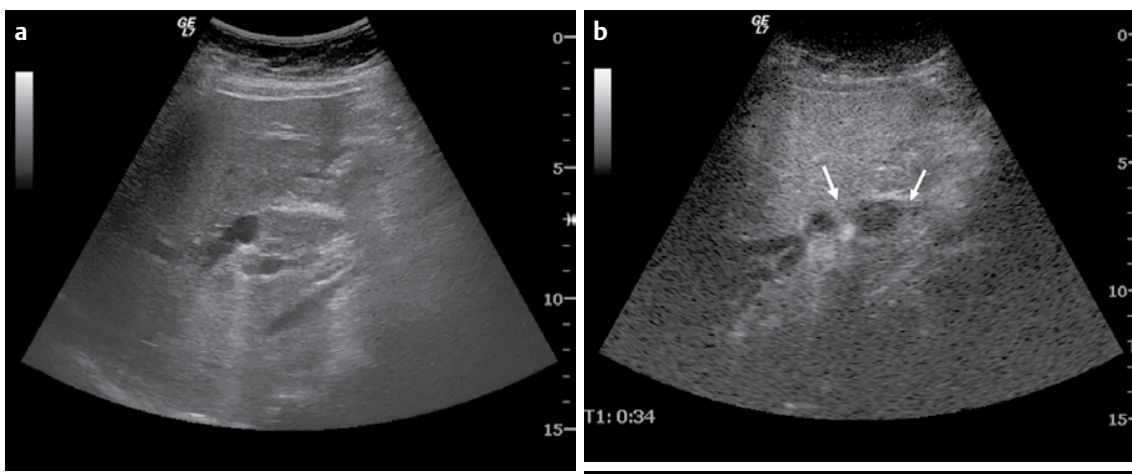

Fig. 3 Extension of extrahepatic cholangiocarcinoma to the liver. Baseline ultrasound shows a dilated common bile duct occupied by an isoechoic cast $\mathbf{a}$. CEUS in the arterial phase $\mathbf{b}$ shows peripheral enhancement of the lesion and washout with extension to the surrounding liver parenchyma in the late phase $\mathbf{c}$. The same finding is visualized on portal phase contrast-enhanced CT d.
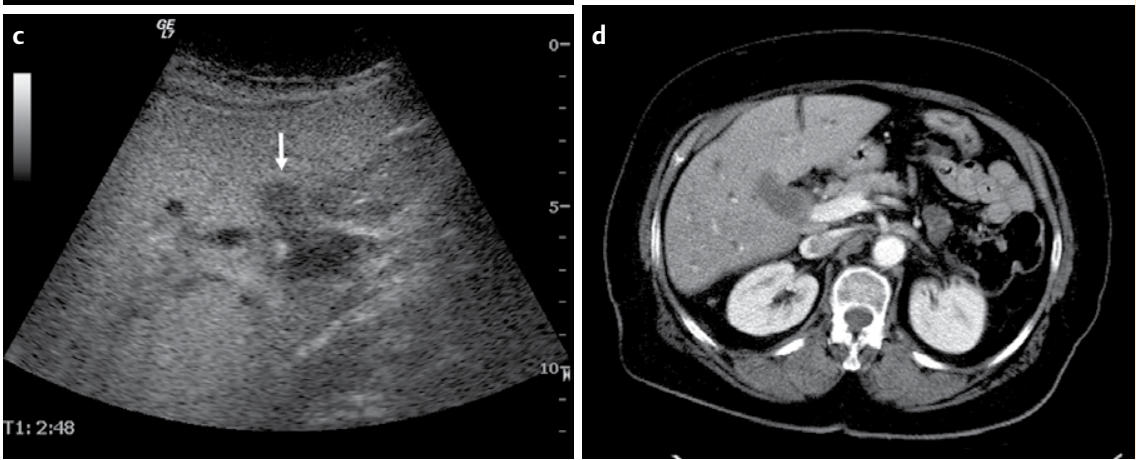

4 patients presented with hepatocellular carcinoma. In 2 cases, CEUS demonstrated malignant infiltration of the bile ducts, presenting with early arterial phase enhancement, followed by washout in the portal and later phases. In 2 other cases, distant metastatic, obstructive biliary lesions with similar behavior to the original tumor in the dynamic study were detected ( $\bullet$ Fig. $\mathbf{5}$ ).
6 cases corresponded to bile duct metastases from gastric carcinoma $(\mathrm{n}=3)$, colonic carcinoma $(\mathrm{n}=2)$ and renal carcinoma $(n=1)$. In 4 cases CEUS demonstrated intraductal lesions in the hilar confluence and extrahepatic biliary ducts, while bile duct infiltration by contiguous liver malignancy was observed in the remaining 2 cases ( 0 Fig. $\mathbf{6}$ ). 

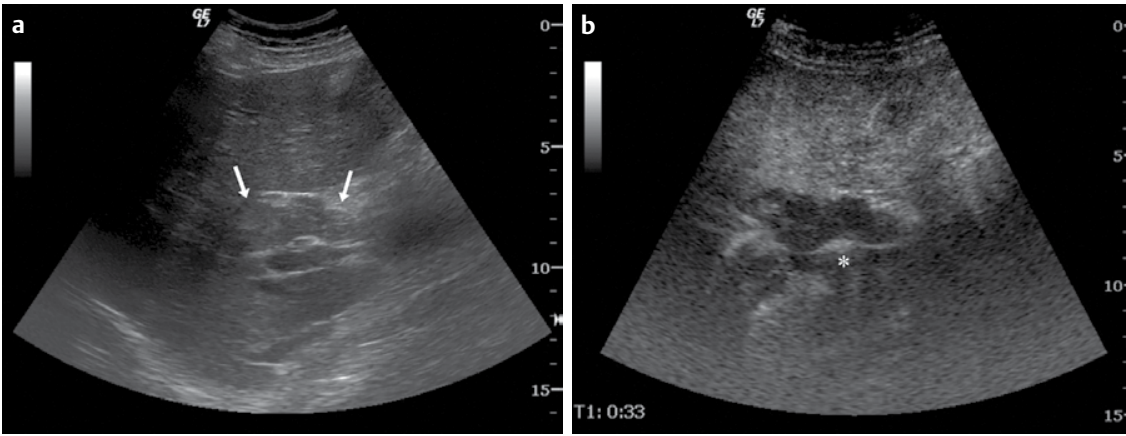

Fig. 4 Necrotic cholangiocarcinoma. Baseline ultrasound a shows a dilated common bile duct filled with intraluminal cast isoechoic to the liver parenchyma (arrows). CEUS b shows the absence of enhancement in the necrotic avascular mass and thrombosed portal vein (asterisk).
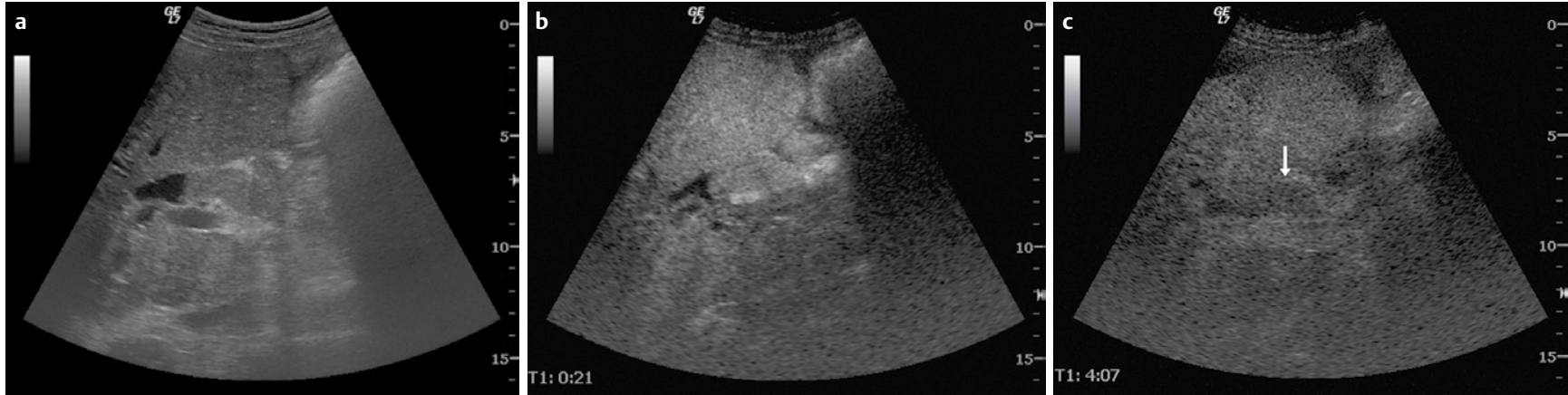

Fig. 5 Metastatic hepatocellular carcinoma to biliary ducts in a patient treated with previous multiple radiofrequency ablations. Baseline ultrasound shows an intraductal mass $\mathbf{a}$. CEUS shows enhancement in the arterial phase $\mathbf{b}$ and late washout that parallels the contrast behavior of the original mass $\mathbf{c}$.
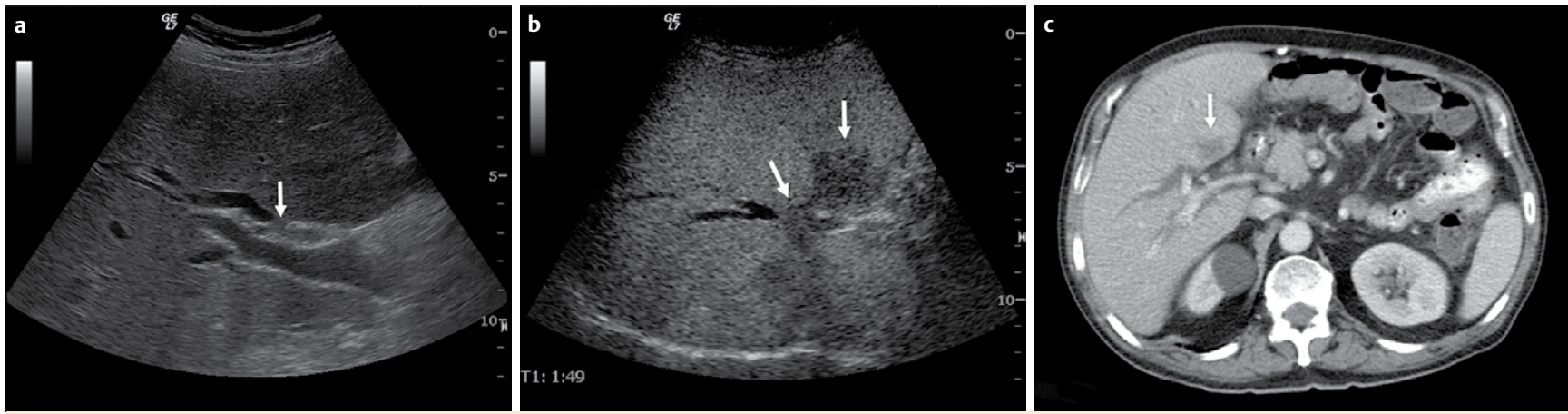

Fig. 6 Spread of liver metastasis to bile duct. Baseline ultrasound a shows mild dilatation of intrahepatic bile ducts and a hyperechoic cast in the common hepatic duct. CEUS in the late phase $\mathbf{b}$ shows washout in a metastasic parenchymatous lesion extending to the bilary duct that is not visualized on portal phase contrast-enhanced CT c.

CEUS identified 3 of 6 cases of pancreatic carcinoma, disclosing a hypoenhancing mass in the arterial phase not visible on baseline ultrasound.

In one case, bile duct obstruction by lymphoma presented as a discrete hypoenhancing mass in the pancreatic head with peripancreatic adenopathies.

Regarding benign lesions, CEUS correctly identified 14 of 15 cases $(93.3 \%)$ corresponding to biliary sludge and stones that presented as intraluminal casts with variable echogenicity on baseline ultrasound, with no enhancement after contrast administration ( $\bullet$ Fig. 7). One case of cholangitis showed diffuse hyperenhancement in the duct wall during the arterial phase and wash-out in the portal and late phase. In another case, baseline ultrasound demonstrated ductal dilatation secondary to a heterogeneous hilar mass, with calcification, extending to the gallbladder and engulfing biliary ducts and vessels. CEUS showed intense enhancement in the arterial phase, with posterior washout. Pathological study disclosed infiltrative xanthogranulomatous cholecystitis ( $\bullet$ Fig. 8).
We also studied 7 patients with suspected biliary prosthesis obstruction. CEUS showed no enhancement in 5 cases ( $\bullet$ Fig. 7), which came out to be sludge, and enhancement in 2 other cases corresponding to cholangiocarcinoma relapse and metastasic hypernephroma.

In 6 cases the results of CEUS were indeterminate. In 4 of these cases, lesions affected the intrapancreatic distal common biliary duct. The location of the lesions in the biliary tree is reflected in $\odot$ Table 3.

CEUS correctly identified 36 out of 42 malignant lesions and 15 of 17 benign lesions, resulting in an overall sensitivity of $85.7 \%$ and a specificity of $88.2 \%$. Its positive predictive value was $94.7 \%$ while the negative predictive value was $71.4 \%$, with an overall diagnostic accuracy of $86.4 \%$ ( $\odot$ Table 4 ).

\section{Discussion}

$\nabla$

Basal ultrasound is the first-line imaging test in patients with a suspected biliary duct obstruction, permitting a differentiation 

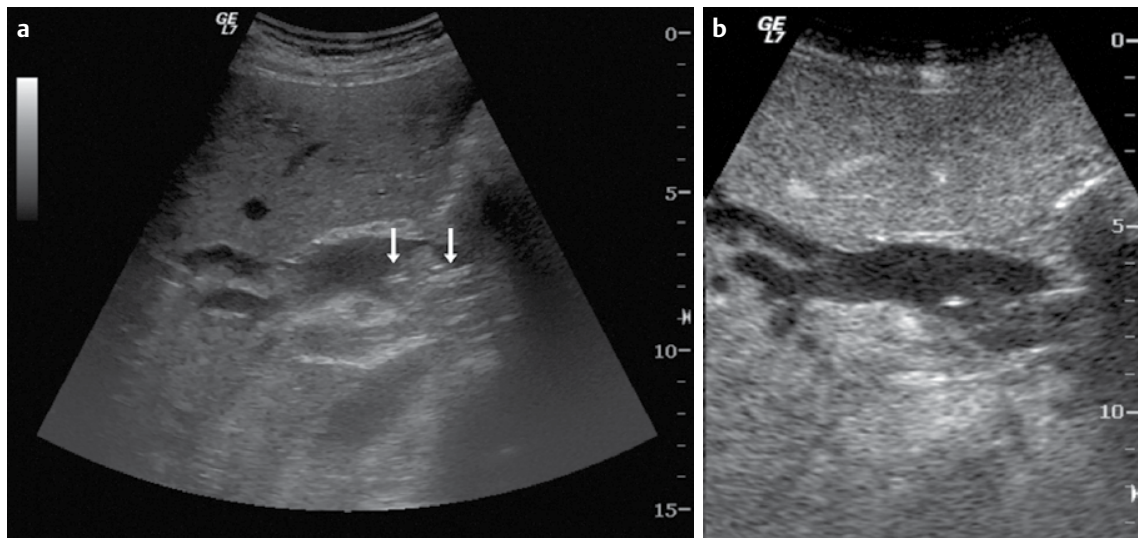

Fig. 7 Biliary sludge in a patient with obstructive cholangiocarcinoma treated with ductal prosthesis. Baseline ultrasound shows stent (arrow) encircled by an echogenic mass a. CEUS shows the absence of enhancement in the mass, confirming its inert nature $\mathbf{b}$.
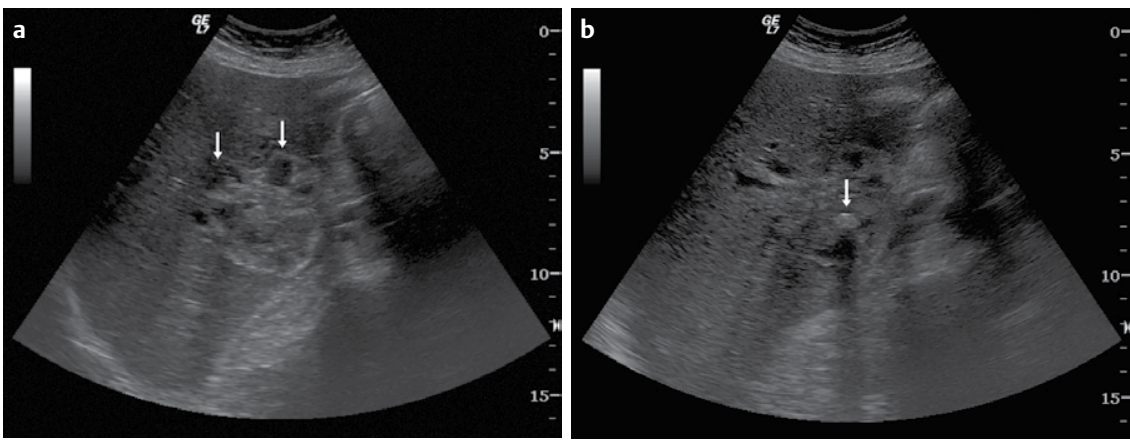

Fig. 8 Xantogranulomatous mass. Baseline ultrasound a shows a heterogeneous, predominantly hyperechoic hilar mass with bile duct dilatation (arrows) and calcification (arrow in b). CEUS shows significant arterial enhancement $\mathbf{c}$ with posterior washout (not shown) that correlates with portal phase contrast-enhanced CT d.
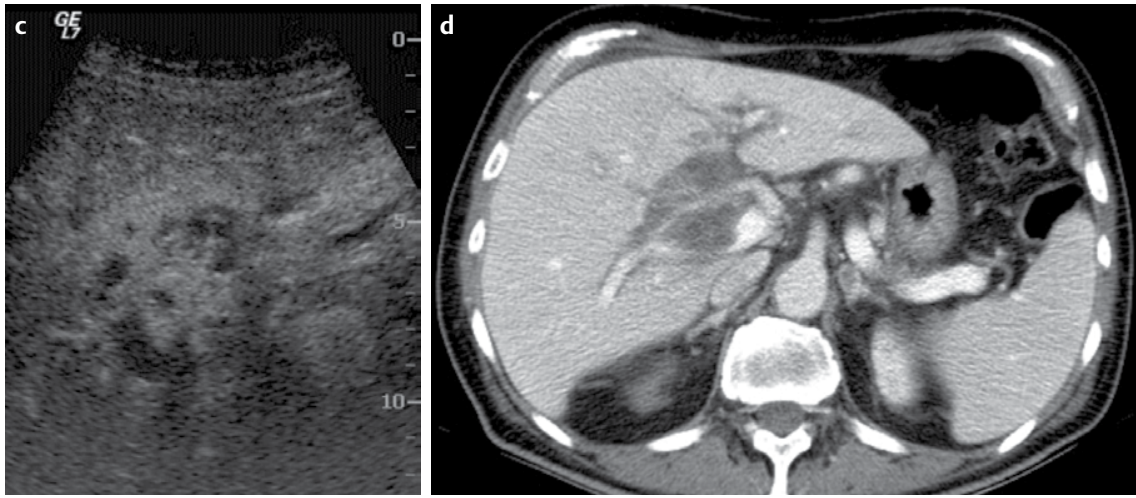

Table 3 Location of lesions in the biliary tree.

\begin{tabular}{|lc|}
\hline Lesion location & Number \\
Intrahepatic & 3 \\
\hline Hilar & 9 \\
\hline Common hepatic duct & 12 \\
\hline Common biliary duct & 17 \\
\hline Intrahepatic & 11 \\
\hline Biliary prosthesis & 7 \\
\hline Total & 59 \\
\hline
\end{tabular}

between intra- and extrahepatic cholestasis. However, this technique offers a low sensitivity at the time of establishing the nature of the lesion. In the present study, we have demonstrated that CEUS improves diagnosis, differentiating benign from malignant lesions in the majority of cases. We believe that CEUS represents an appropriate first-line diagnostic procedure following initial identification of a biliary obstruction by non-enhanced ultrasound allowing segregation of patients who require CT or MR for staging and surgical planning from those who can proceed directly to ERCP. Performing CEUS during the same session can save time and is financially prudent.

\begin{tabular}{|c|c|c|c|}
\hline \multirow[t]{2}{*}{ CEUS } & \multicolumn{3}{|c|}{ Pathologic result/CT/MR/ERCP } \\
\hline & Malignant & Benign & Total \\
\hline Malignant & 36 & 2 & 38 \\
\hline \multirow[t]{2}{*}{ Benign } & 6 & 15 & 21 \\
\hline & 42 & 17 & 59 \\
\hline
\end{tabular}

The comparison of CEUS and contrast-enhanced cross-sectional imaging is sometimes problematic. CEUS offers real-time dynamic study without gaps in data acquisition, while CT and MR have scan delays and, consequently, may miss the early initial enhancement in the arterial phase. Moreover, the use of a purely intravascular contrast medium can avoid some problematic effects happening with the interstitial diffusion in MR and CT contrast agents, such as masquerading of some lesions during the portal and late phase produced by the persistent enhancement [10]. Alternatively the lack of interstitial enhancement in CEUS can theoretically limit differentiation between neoplastic processes with or without an important interstitial component. 
In CEUS images, intrahepatic bile duct dilation is more conspicuous, due to parenchymal enhancement, improving the detection of intraluminal lesions, whether malignant or corresponding to sludge or biliary stones. In patients with neoplastic jaundice, ascertaining exactly the cause, level and extension of the lesion is necessary, in order to select candidates for surgical resection. Similarly to CT and MR, CEUS allows a more precise determination of neoplasic parenchymatous extension in tumors of ductal origin, as also ductal extension of lesions originated in the liver parenchyma.

Cholangiocarcinoma is the most frequent biliary neoplasm, classified as intrahepatic (peripheral and hilar) or extrahepatic. Hilar cholangiocarcinoma originates in the bile duct bifurcation, and tends to infiltrate the adjacent liver parenchyma. Basal ultrasound shows intrahepatic dilatation with a normal extrahepatic duct, but has a low capacity to establish the nature of the obstruction, due to the frequent isoechogenicity of these lesions with normal liver parenchyma and its infiltrative nature [7-9]. In our experience, Klatskin tumors show a variable enhancement in early phases with a constant washout in late phases which nicely delineates the limits of the lesion. These features are consistent with those described in the literature. $\mathrm{Xu}$ et al. [11] studied the dynamic behavior of hilar cholangiocarcinoma in CEUS, demonstrating hyperenhancement, isoenhancement and hypoenhancement in the arterial phase in 43.8, 43.8 and $12.6 \%$ of cases, respectively, with a ring, homogeneous enhancement pattern or heterogeneous enhancement pattern in $9.4,34.4$ and $56.2 \%$ of cases, respectively. In the portal and late phases, $93.8 \%$ of the cases presented washout, which improved the delimitation of the tumor margins.

Extrahepatic cholangiocarcinoma can also show variable enhancement in the CEUS arterial phase, with washout in late phases in most cases. The essential contribution of CEUS is differentiation among tumors and non-enhancing, inert material, including lithiasis without acoustic shadow, blood clots or detritus $[7,10,12]$.

Bile duct infiltration is a rare complication of hepatocellular carcinomas and can present with contiguous direct invasion or embolization of an occult parenchymatous lesion [9]. Both presentations were observed in this study, with an enhancement pattern similar to the original tumor, hyperenhancement in the arterial phase and contrast wash-out in the late phase [13]. CEUS is also useful to categorize intrabiliary metastasic lesions of a non-hepatic origin. These are infrequent lesions with a difficult differential diagnosis with malignant primary lesions. They can present as fully intraductal lesions or as secondary invasive lesions from adjacent liver metastases. Different primary tumors can cause biliary metastatic obstruction but gastrointestinal malignancies, particularly colonic cancer, are most frequent [9]. In our study, gastric cancer was most common.

CEUS improves the detection of pancreatic head tumors not detected by basal ultrasound. The pancreas enhances homogeneously in the arterial phase and presents a transient venous phase, facilitating detection of carcinoma, which presents typically with hypoenhancement in all phases [6]. Fan et al. [14] reported a sensitivity, specificity and accuracy of 91.7, 97.2 and $87 \%$, respectively, at the time of detection of pancreatic adenocarcinomas with CEUS. The results are similar to those obtained with contrast-enhanced CT.
Benign lesions show no enhancement on CEUS, due to the inert nature of intraductal contents (biliary sludge, lithiasis, blood clots or detritus), allowing differentiation from tumoral casts in most cases.

Even if no enhancement is the rule in benign lesions, there are exceptions, which include inflammatory conditions such as xanthogranulomatous lesions or the faint captation of the ductal wall in cases of cholangitis. These examples emphasize that enhancement is not synonymous with malignant lesion. Alternatively, extensive necrotic malignant lesions can present without contrast enhancement in rare instances.

CEUS shares some limitations with basal ultrasound, including operator dependence. Obesity and bowel gas are important obstacles in some cases, particularly during the evaluation of the distal bile duct. This limitation was behind most indeterminate cases in this study.

Important limitations of the study are its retrospective design and the absence of a unitary reference test, due to the impossibility of obtaining a pathologic diagnosis in some cases. In the latter cases we used CT, MR and follow-up to set a reference diagnosis. Prospective studies are necessary to validate our conclusions, as are comparative investigations about agreement among CEUS, on one side, and CT or MR, on the other.

\section{Conclusion \\ $\nabla$}

CEUS is useful to differentiate the benign or malignant nature of a biliary duct obstruction. It may offer a combined approach with CT and MR, saving the indication of these techniques in unequivocal cases of benignity and represents an alternative in those cases in which these techniques are hazardous or contraindicated, including instances of contrast allergy or kidney failure. In addition, CEUS is able to disclose tumoral invasion of biliary ducts by liver tumors and helps to define the local extension of hilar hepatobiliary neoplasms.

\section{References}

1 Rogoveanu I, Gheonea DI, Saftoiu A et al. The role of imaging methods in identifying the causes of extrahepatic cholestasis. J Gastrointestin Liver Dis 2006; 15: 265-271

2 Blackbourne LH, Earnhardt RC, Sistrom CL et al. The sensitivity and role of ultrasound in the evaluation of biliary obstruction. Am Surg 1994; 60: 683-690

3 Wilson SR, Burns PN. Microbubble-enhanced US in Body Imaging: What Role? Radiology 2010; 257: 24-39

4 Piscaglia F, Bolondi L. The safety of Sonovue in abdominal applications: retrospective analysis of 23188 investigations. Ultrasound Med Biol 2006; 32: 1369-1375

5 Claudon M, Dietrich CF, Choi BI et al. Guidelines and good clinical practice recommendations for contrast enhanced ultrasound (CEUS) in the liver - update 2012: a WFUMB-EFSUMB initiative in cooperation with representatives of AFSUMB, AIUM, ASUM, FLAUS and ICUS. Ultraschall Med 2013; 34: 11-29

6 Piscaglia F, Nolsøe C, Dietrich CF et al. The EFSUMB Guidelines and Recommendations on the Clinical Practice of Contrast Enhanced Ultrasound (CEUS): update 2011 on non-hepatic applications. Ultraschall Med 2012; 33: 33-59

$7 \mathrm{Xu} \mathrm{H-X.Contrast-enhanced} \mathrm{ultrasound} \mathrm{in} \mathrm{the} \mathrm{biliary} \mathrm{system:} \mathrm{Potential}$ uses and indications. World J Radiol 2009; 1: 37-44

8 Meacock LM, Sellars ME, Sidhu PS. Evaluation of gallbladder and biliary duct disease using microbubble contrast-enhanced ultrasound. $\mathrm{Br} \mathrm{J}$ Radiol 2010; 83: 615-627

9 Sparchez Z. Role of contrast enhanced ultrasound in the assessment of biliary duct disease. Med Ultrason 2014; 16: 41-47 


\section{E18 Original Article}

10 Dietrich $C F$, Cui XW, Boozari B et al. Contrast-enhanced ultrasound (CEUS) in the diagnostic algorithm of hepatocellular and cholangiocellular carcinoma, comments on the AASLD guidelines. Ultraschall Med 2012; 33: S57-S66

$11 \mathrm{Xu} H$-X, Chen L-D, Xie X-Y et al. Enhancement pattern of hilar cholangiocarcinoma: contrast-enhanced ultrasound versus contrast-enhanced computed tomography. Eur J Radiol 2010; 75: 197-202

12 Liu LN, Xu HX, Zheng SG et al. Ultrasound Findings of Intraductal Papillary Neoplasm in Bile Duct and the Added Value of Contrast-Enhanced Ultrasound. Ultraschall Med 2014; DOI: 10.1055/s-0034-1366672
13 Wildner D, Bernatik T, Greis C et al. CEUS in Hepatocellular Carcinoma and Intrahepatic Cholangiocellular Carcinoma in 320. Patients-Early or Late Washout Matters: A Subanalysis of the DEGUM Multicenter Trial. Ultraschall Med 2015; 36: 132-139

14 Fan Z, Li Y, Yan K et al. Application of contrast-enhanced ultrasound in the diagnosis of solid pancreatic lesions - A comparison of conventional ultrasound and contrast-enhanced CT. Eur J Radiol 2013; 82: 1385-1390 